\title{
Genome-wide analysis reveals prognostic value of novel signature CLU for malignant meningioma patients based on large-scale cohorts
}

\author{
WangRui Liu ${ }^{1}$, Hu Xiaoxin ${ }^{2}$, Jun Wang ${ }^{3}$, Haineng Huang ${ }^{4}$, Kui Chen ${ }^{1}$, yuanyuan $\mathrm{Qu}^{2}$, \\ chuanyu $\mathrm{li}^{4}$, chunguang $\mathrm{ma}^{2}$, wenhao $\mathrm{xu}^{2}$, huadong huang ${ }^{4}$, and Chunlong Zhong ${ }^{1}$ \\ ${ }^{1}$ Shanghai East Hospital \\ ${ }^{2}$ Fudan University Shanghai Cancer Center \\ ${ }^{3}$ Sun Yat-sen University Cancer Center \\ ${ }^{4}$ Youjiang Medical University for Nationalities
}

November 4, 2020

\begin{abstract}
Background: Meningioma is one of the most common tumors of central nervous system. Although genetic alterations have been linked to elevated risk of malignancy for meningioma patients, the incongruence between clinical outcomes and WHO grade classification still exist. Therefore, large-scale genome-wide expression profiles identifying reliable biomarkers remains incompletely investigated. Methods: In order to identify genes related to invasion and metastasis process in meningiomas, genome-wide profiles in 145 meningioma patients were identified using microarray datasets GSE12530, GSE16581 and GSE43290 from the Gene Expression Synthesis (GEO) database based on differential tissues expression. Protein-protein interaction (PPI) network were constructed and functional annotations of DEGs were evaluated using $\mathrm{R}$ software. In addition, differential expression and prognostic value of CLU on meningioma was evaluated using immunohistochemistry in WHO grade II-III meningioma from AHYMUN, a real-world cohort. Results: A total of 58 DEGs were significantly associated with malignant behaviours of meningioma. Next, CLU were identified as hub gene in the PPI network, showing markedly prognostic implications in 67 meningioma patients. Expression level of CLU significantly climbed with elevated WHO risk classification. Interestingly, 40 genes were screened according to differential CLU expression. Additionally, significant high expression level of CLU were found in meningiomas tissue than normal tissues, predicting significant poor prognosis for 100 meningiomas patients from AHYMUN cohort. Conclusion: In conclusion, this study first reveals novel role of CLU using genome-wide expression profiles in high-risk WHO grade of meningioma and significant prognostic value based on large-scale cohorts. This work laid the foundation for the targeted molecular therapies and provided new insights into the treatment and prognostic srategies of meningiomas.
\end{abstract}

\section{Hosted file}

Manuscript.DOCX available at https://authorea.com/users/373073/articles/490864-genomewide-analysis-reveals-prognostic-value-of-novel-signature-clu-for-malignant-meningiomapatients-based-on-large-scale-cohorts

\section{Hosted file}

Table 1.pdf available at https://authorea.com/users/373073/articles/490864-genome-wideanalysis-reveals-prognostic-value-of-novel-signature-clu-for-malignant-meningiomapatients-based-on-large-scale-cohorts

\section{Hosted file}


Table 2.pdf available at https://authorea.com/users/373073/articles/490864-genome-wideanalysis-reveals-prognostic-value-of-novel-signature-clu-for-malignant-meningiomapatients-based-on-large-scale-cohorts

\section{Hosted file}

Table 3.pdf available at https://authorea.com/users/373073/articles/490864-genome-wideanalysis-reveals-prognostic-value-of-novel-signature-clu-for-malignant-meningiomapatients-based-on-large-scale-cohorts

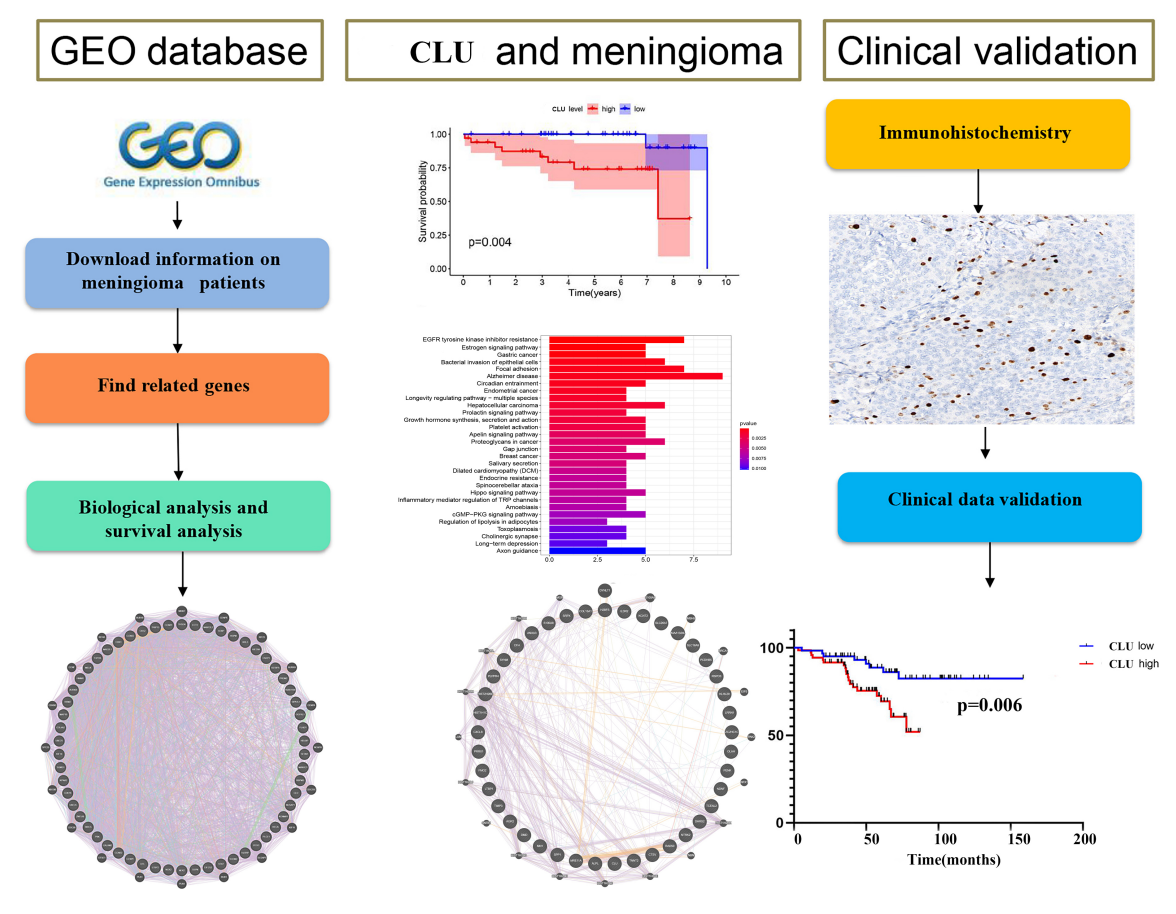

$\mathbf{A}$

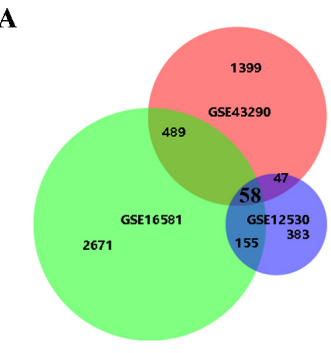

D

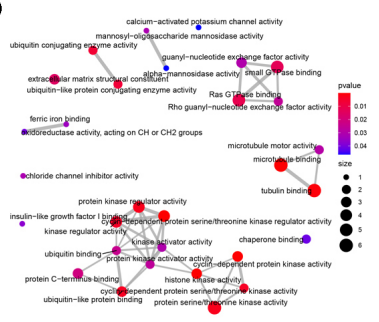

B

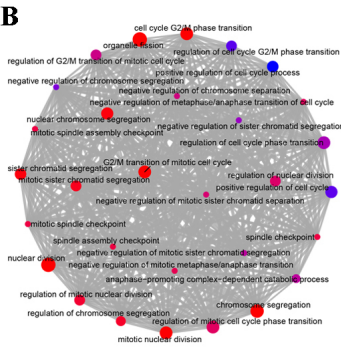

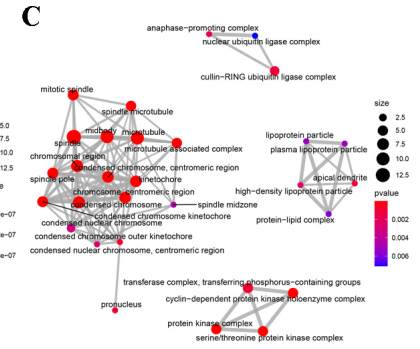

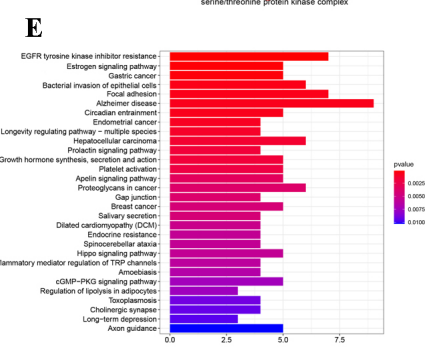




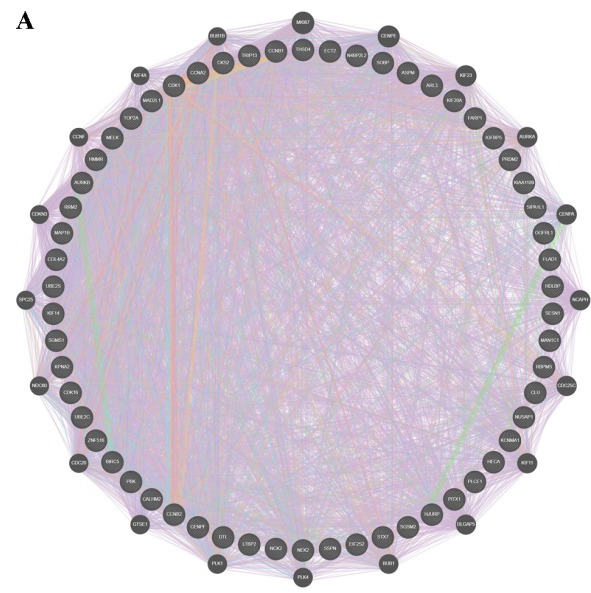

B

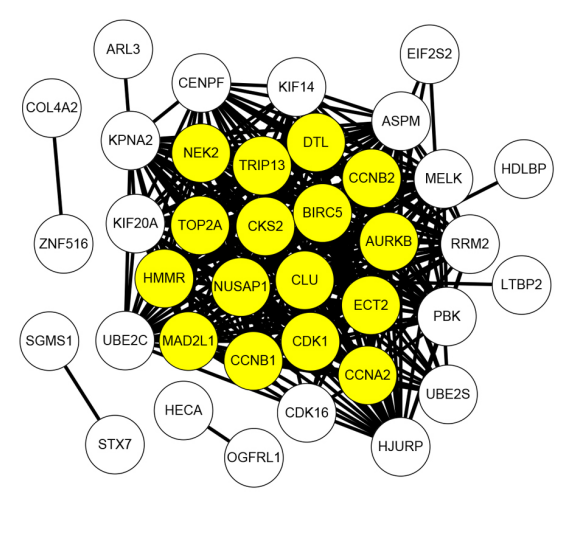

A

B pvalue Hazard ratio ।

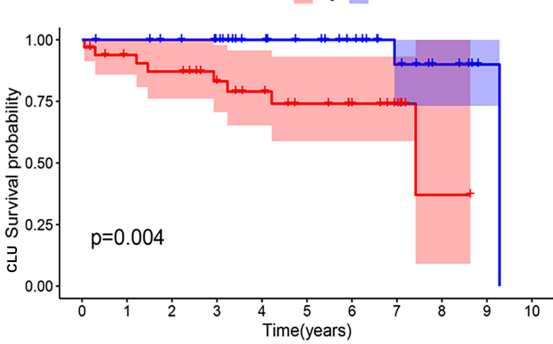

CLU $\quad 0.0219 .291(0.698-123.633)$

Grade $\quad 0.019 \quad 3.193(1.207-8.447)$

Gender $0.306 \quad 0.439(0.091-2.124)$

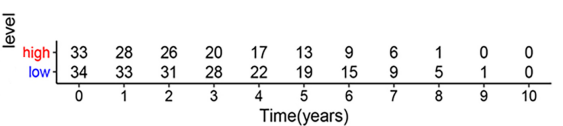

Age $\quad 0.059 \quad 1.058(0.998-1.122)$

C pva

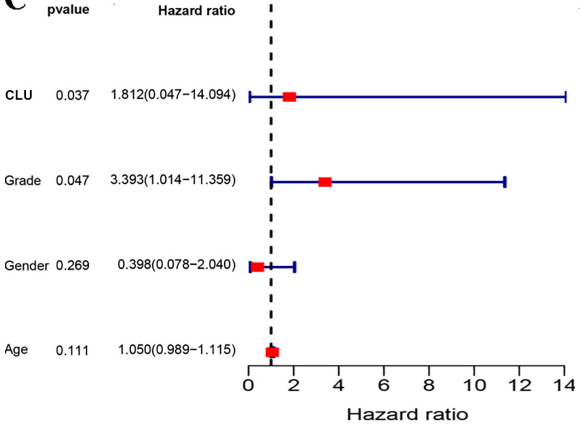

D

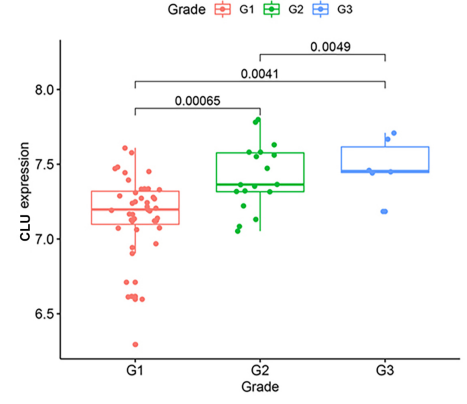

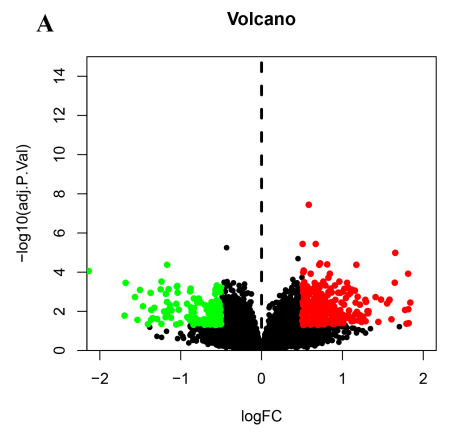

B

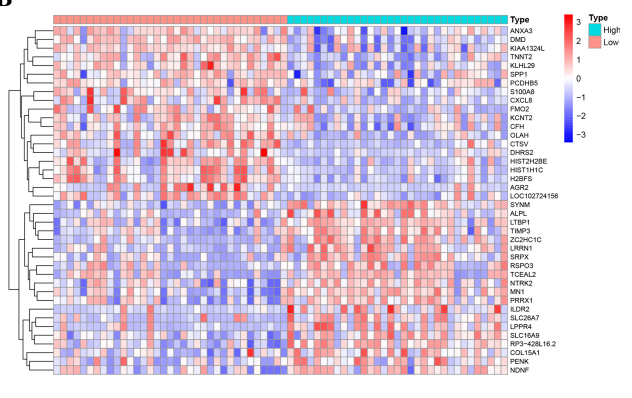


A

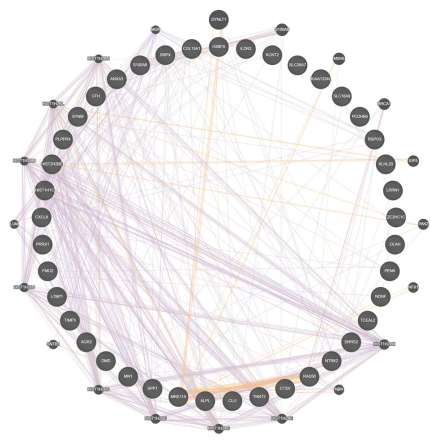

C

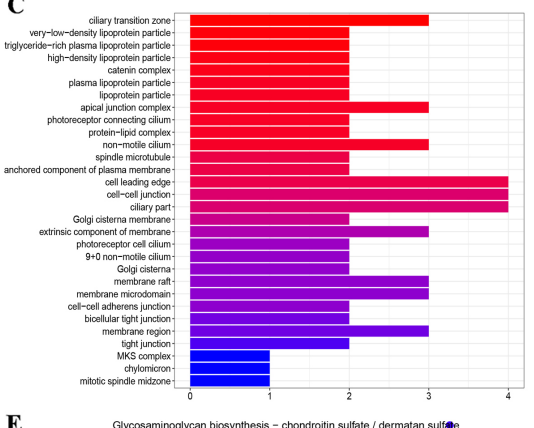

E

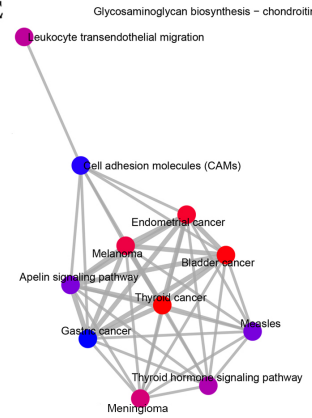

B
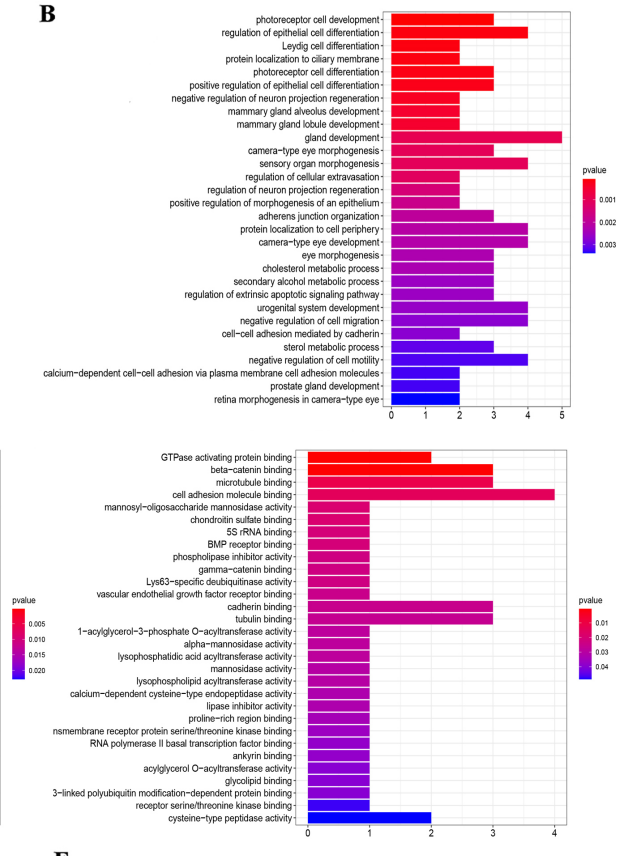

F

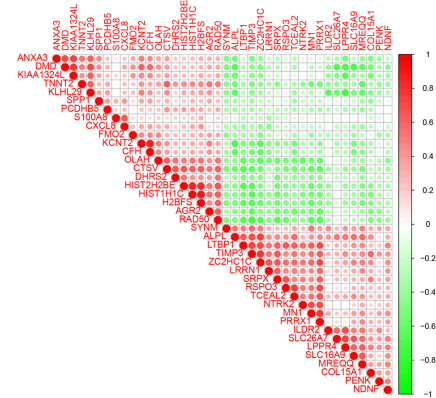



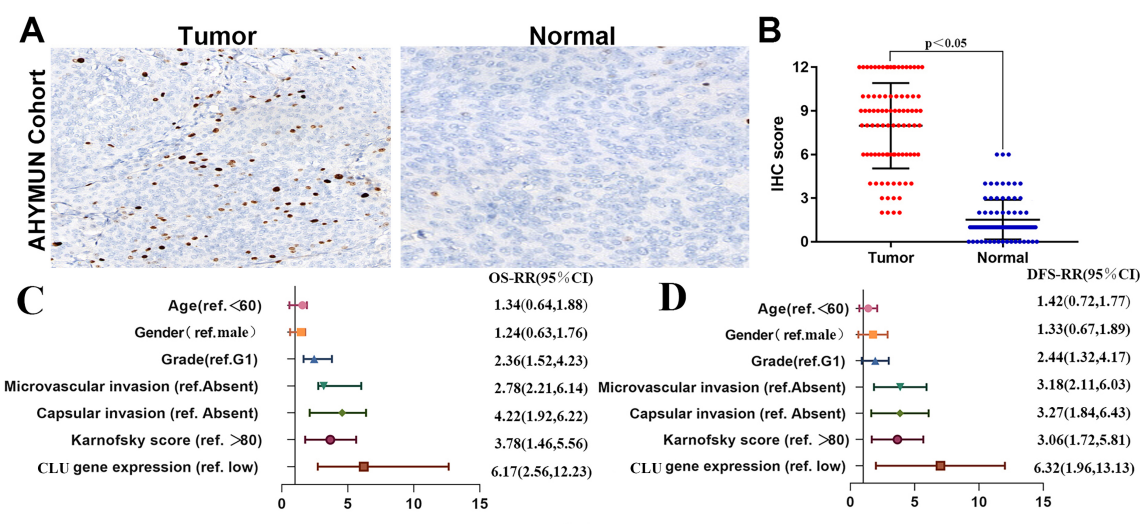

OS-RR $(95 \% \mathrm{CI})$ $134(0.64,188)$ $1.24(0.63,1.76)$ 2.36(1.52,4.23) 2.78(2.21,6.14) Microvascular invasion (refeAbsent) 4.22(1.92,6.22) Capsular invasion (ref. Absent) 3.78(1.46,5.56) Karnofsky score (ref. $>80)-\longmapsto \quad 3.06(1.72,5.81)$ 6.17(2.56,12.23) CLU gene expression (ref. low)

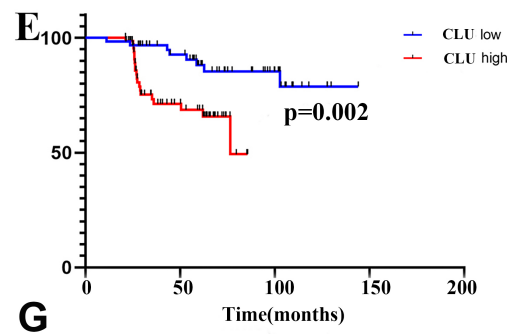

F
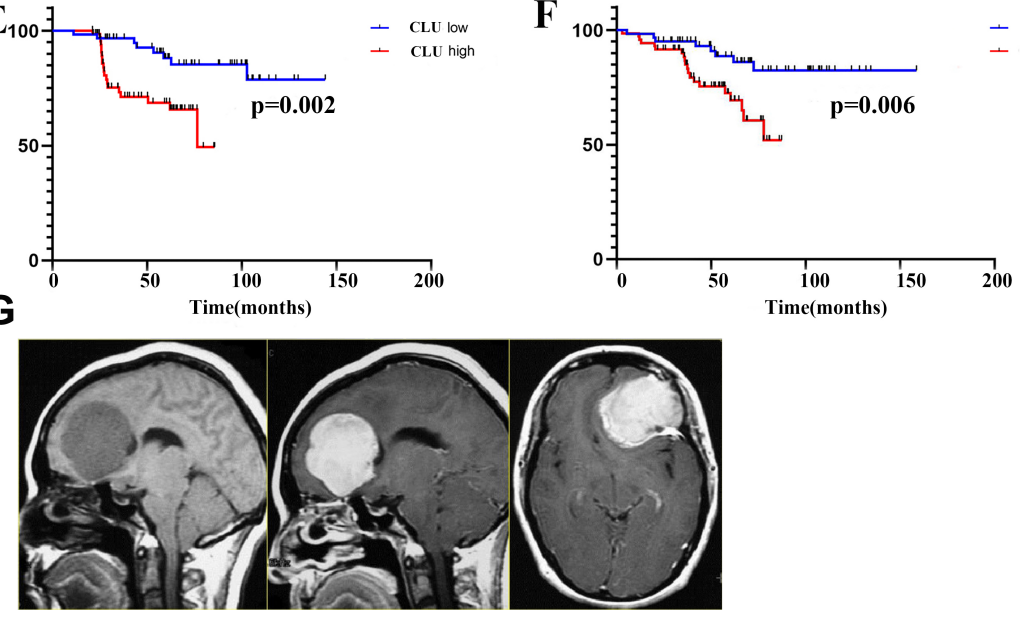